University of Nebraska - Lincoln

DigitalCommons@University of Nebraska - Lincoln

USDA National Wildlife Research Center - Staff Publications
U.S. Department of Agriculture: Animal and Plant Health Inspection Service

2009

\title{
Eight Polymorphic Microsatellite Loci Developed and Characterized From Townsend's Big-Eared Bat, Corynorhinus Townsendii
}

\author{
Antoinette J. Piaggio \\ USDA/APHIS/WS National Wildlife Research Center, Toni.J.Piaggio@aphis.usda.gov \\ Katie Erin G. Miller \\ Idaho State University, Department of Biological Sciences \\ Marjorie D. Matocq \\ Idaho State University, Department of Biological Sciences \\ Susan L. Perkins \\ Division of Invertebrates, American Museum of Natural History
}

Follow this and additional works at: https://digitalcommons.unl.edu/icwdm_usdanwrc

Part of the Environmental Sciences Commons

\footnotetext{
Piaggio, Antoinette J.; Miller, Katie Erin G.; Matocq, Marjorie D.; and Perkins, Susan L., "Eight Polymorphic Microsatellite Loci Developed and Characterized From Townsend's Big-Eared Bat, Corynorhinus Townsendii" (2009). USDA National Wildlife Research Center - Staff Publications. 951. https://digitalcommons.unl.edu/icwdm_usdanwrc/951
}

This Article is brought to you for free and open access by the U.S. Department of Agriculture: Animal and Plant Health Inspection Service at DigitalCommons@University of Nebraska - Lincoln. It has been accepted for inclusion in USDA National Wildlife Research Center - Staff Publications by an authorized administrator of DigitalCommons@University of Nebraska - Lincoln. 


\title{
PERMANENT GENETIC RESOURCES Eight polymorphic microsatellite loci developed and characterized from Townsend's big-eared bat, Corynorhinus townsendii
}

\author{
ANTOINETTE J. PIAGGIO,${ }^{*}$ KATIE ERIN G. MilleR,+MARJORIE D. MATOCQ† and \\ SUSAN L. PERKINS \\ *Wildlife Services, National Wildlife Research Center, Wildlife Genetics Laboratory, US Department of Agriculture, 4101 LaPorte \\ Avenue, Fort Collins, CO 80521, USA, +Department of Biological Sciences, Idaho State University, Campus Box 8007 Pocatello, \\ ID 83209-8007, USA, †Division of Invertebrates, American Museum of Natural History, Central Park West at 79th Street, New York, \\ NY 10024, USA
}

\begin{abstract}
Two of the five subspecies of the western big-eared bat, Corynorhinus townsendii, are listed as federally endangered with the remaining three being of conservation concern. Knowing the degree of connectivity among populations would aid in the establishment of sound conservation and management plans for this taxon. For this purpose, we have developed and characterized eight polymorphic microsatellite markers.
\end{abstract}

Keywords: Corynorhinus townsendii, microsatellite, Townsend's big-eared bat

Received 16 March 2008; revision received 18 April 2008; revision accepted 30 April 2008

Townsend's big-eared bat, Corynorhinus townsendii, is a North American bat of the family Vespertilionidae. There are five subspecies in the USA and Mexico (Piaggio \& Perkins 2005) and two, Corynorhinus townsendii townsendii and Corynorhinus townsendii pallescens, are listed as species of Special Concern or sensitive species by state and federal agencies (Pierson et al. 1999), while Corynorhinus townsendii ingens and Corynorhinus townsendii virginianus are federally listed as Endangered. Although many local, state and federal agencies have developed management plans for C. townsendii that include monitoring and protection, little is known about their population structure or connectivity.

Population-level genetic data would significantly increase our understanding of $C$. townsendii. Only five of 15 microsatellite primers designed for other microchiropteran species (Burland et al. 1998; Vonhof et al. 2002) amplified and were variable in C. townsendii (Piaggio et al. in press). To increase the number of markers for this species, we developed and characterized eight new microsatellite loci.

Tissue samples were obtained from 25 individuals from Colorado (C.t. pallescens) and from 29 individuals from

Correspondence: Antoinette J. Piaggio, USDA/APHIS/WS/ National Wildlife Research Center, Wildlife Genetics Laboratory, 4101 LaPorte Avenue, Fort Collins, CO 80521, USA. Fax: (970) 266 6063; E-mail: toni.j.piaggio@aphis.usda.gov
Idaho (C. t. townsendii). One individual from Colorado was used in the enrichment and development process. We targeted these localities because most current populationlevel research of $C$. townsendii is focused on these subspecies. We developed a microsatellite library following methods adapted from Glenn \& Schable (2005). Genomic DNA was digested with the enzyme $R s a \mathrm{I}$ and fragments were ligated using double-stranded SNX-24 linkers. This library was hybridized to 12 biotinylated microsatellite oligonucleotide probes with dinucleotide and trinucleotide repeats (e.g., GT, CA, TG, CAC and CAG). Hybridized fragments were captured on streptavidin-coated Dynabeads (Dynal Biotech). Microsatellite-enriched fragments were amplified and cloned with the TOPO TA cloning kit (Invitrogen). Insert sequences from 96 colonies were obtained with M13 forward and reverse primers and visualized on an ABI 3730xl genetic analyser (Applied Biosystems). Forty-one clones had recognizable microsatellite sequences, of which $88 \%$ (36) had adequate flanking regions to design primers, which was accomplished with Staden package (Staden et al. 1998), TROLL (Castelo et al. 2002; Martins et al. 2006), and web-based Primer 3 (Rozen \& Skaletsky 2000) software packages.

Polymerase chain reactions (PCR) were carried out using $0.5 \mu \mathrm{L}$ each of $10 \mu \mathrm{M} 5^{\prime}$ fluorescently end-labelled primers (Table 1), $3.0 \mu \mathrm{L}$ nanopure water, $5.0 \mu \mathrm{L}$ ReddyMix (ABgene), and $1.0 \mu \mathrm{L}$ of DNA (6-25 ng DNA/ $\mu \mathrm{L})$. The thermal profile 
Table 1 Characteristics of the eight microsatellite loci that were developed and optimized from Corynorhinus townsendii

\begin{tabular}{|c|c|c|c|c|c|c|c|c|}
\hline $\begin{array}{l}\text { Locus/primer } \\
\text { name }\end{array}$ & $\begin{array}{l}\text { Primer sequence }\left(5^{\prime}-3^{\prime}\right) ; \mathrm{F} \text {, forward; } \\
\mathrm{R} \text {, reverset }\end{array}$ & $\begin{array}{l}\text { Repeat } \\
\text { motif }\end{array}$ & $\begin{array}{l}\text { Size range } \\
(\mathrm{bp})\end{array}$ & $\begin{array}{l}N_{\text {A }} \text { per } \\
\text { population }\end{array}$ & $\begin{array}{l}N_{\mathrm{A}} \\
\text { Total }\end{array}$ & $H_{\mathrm{O}}$ & $H_{\mathrm{E}}$ & $\begin{array}{l}\text { Null allele } \\
\text { freqs. }\end{array}$ \\
\hline Coto_B02F_B02R & $\begin{array}{l}\text { F: NED-CCAGCTAGAAGTTGAGAGTCAGA } \\
\text { R: GTCTCTTGTCACACTTTCTGTCC }\end{array}$ & $(\mathrm{TC})_{14}(\mathrm{AC})_{12}$ & $\begin{array}{l}\text { CO:150-206 } \\
\text { ID:152-184 }\end{array}$ & $\begin{array}{l}\text { CO:14 } \\
\text { ID:13 }\end{array}$ & 18 & $\begin{array}{l}\text { CO: } 0.88 \\
\text { ID:0.86 }\end{array}$ & $\begin{array}{l}\text { CO:0.85 } \\
\text { ID:0.91 }\end{array}$ & $\begin{array}{l}\text { CO:0.000 } \\
\text { ID:0.0154 }\end{array}$ \\
\hline Coto_G07F_G07R & $\begin{array}{l}\text { F: HEX-GATGAAGATTCAGCTTATGATGC } \\
\text { R: AGCCCTCTATTTCATACCACAGT }\end{array}$ & $(\mathrm{GT})_{9}$ & $\begin{array}{l}\text { CO:314-324 } \\
\text { ID:316-324 }\end{array}$ & $\begin{array}{l}\text { CO:6 } \\
\text { ID:4 }\end{array}$ & 6 & $\begin{array}{l}\text { CO: } 0.52 \\
\text { ID: } 0.45\end{array}$ & $\begin{array}{l}\text { CO: } 0.64 \\
\text { ID: } 0.43\end{array}$ & $\begin{array}{l}\text { CO:0.063 } \\
\text { ID:0.000 }\end{array}$ \\
\hline Coto_C02F_H08R & $\begin{array}{l}\text { F: FAM-CACCCAGTTGAGAACTATTTGAC } \\
\text { R: TTGAAGGGACTAAATGAACTGAA }\end{array}$ & $(\mathrm{GT})_{24}$ & $\begin{array}{l}\text { CO:171-193 } \\
\text { ID:185-195 }\end{array}$ & $\begin{array}{l}\text { CO:7 } \\
\text { ID:6 }\end{array}$ & 8 & $\begin{array}{l}\text { CO: } 0.76 \\
\text { ID: } 0.38\end{array}$ & $\begin{array}{l}\text { CO: } 0.79 \\
\text { ID: } 0.66^{*}\end{array}$ & $\begin{array}{l}\text { CO:0.009 } \\
\text { ID:0.162§ }\end{array}$ \\
\hline Coto_G12F_B11R & $\begin{array}{l}\text { F: HEX-TGCAAGTCTTAACTCACCTCATT } \\
\text { R: CCACTCCCCTAGTTTTCATCTAC }\end{array}$ & $(\mathrm{AC})_{23}$ & $\begin{array}{l}\text { CO:236-296 } \\
\text { ID:238-268 }\end{array}$ & $\begin{array}{l}\text { CO:17 } \\
\text { ID:11 }\end{array}$ & 20 & $\begin{array}{l}\text { CO: } 0.76 \\
\text { ID:0.86 }\end{array}$ & $\begin{array}{l}\text { CO: } 0.92^{*} \\
\text { ID: } 0.87\end{array}$ & $\begin{array}{l}\text { CO:0.076§ } \\
\text { ID:0.000 }\end{array}$ \\
\hline Coto_H10F_E11R & $\begin{array}{l}\text { F: FAM-AGGCAAACTTTCTTACAGTTGA } \\
\text { TCTTCTTCCATTTTCCTTCAC }\end{array}$ & $(\mathrm{GT})_{20}$ & $\begin{array}{l}\text { CO:242-282 } \\
\text { ID:250-272 }\end{array}$ & $\begin{array}{l}\text { CO:13 } \\
\text { ID:7 }\end{array}$ & 13 & $\begin{array}{l}\text { CO: } 0.72 \\
\text { ID: } 0.48\end{array}$ & $\begin{array}{l}\text { CO: } 0.87^{*} \\
\text { ID: } 0.77^{*}\end{array}$ & $\begin{array}{l}\text { CO:0.072 } \\
\text { ID:0.157§ }\end{array}$ \\
\hline Coto_E09F_B10R & $\begin{array}{l}\text { F: HEX-СТАСССТTССТСТСТTСТTTCTG } \\
\text { R: АТTTСТСССТАТСТССАТСАСТC }\end{array}$ & $(\mathrm{TG})_{20}(\mathrm{GA})_{13}$ & $\begin{array}{l}\text { CO:191-235 } \\
\text { ID:203-229 }\end{array}$ & $\begin{array}{l}\text { CO:13 } \\
\text { ID:11 }\end{array}$ & 16 & $\begin{array}{l}\text { CO:0.96 } \\
\text { ID:0.76 }\end{array}$ & $\begin{array}{l}\text { CO: } 0.88 \\
\text { ID: } 0.86\end{array}$ & $\begin{array}{l}\text { CO:0.000 } \\
\text { ID:0.056 }\end{array}$ \\
\hline Coto_F09F_F10R & $\begin{array}{l}\text { F: FAM-GAGAAGGAAGAGAAACTGGTGTT } \\
\text { R: TACTAAAGAACCTTGACAGTGGC }\end{array}$ & $(\mathrm{AC})_{23}$ & $\begin{array}{l}\text { CO:192-222 } \\
\text { ID:192-220 }\end{array}$ & $\begin{array}{l}\text { CO:10 } \\
\text { ID:11 }\end{array}$ & 12 & $\begin{array}{l}\text { CO:0.64 } \\
\text { ID:0.83 }\end{array}$ & $\begin{array}{l}\text { CO: } 0.84 \\
\text { ID: } 0.88\end{array}$ & $\begin{array}{l}\text { CO:0.101§ } \\
\text { ID:0.020 }\end{array}$ \\
\hline Coto_G02F_H10R & $\begin{array}{l}\text { F: FAM-AGAGTGCTTTTATGGGCAAAT } \\
\text { R: TGCTTGTAGTTCCCTTTCCTT }\end{array}$ & $(\mathrm{GT})_{20}$ & $\begin{array}{l}\text { CO:188-208 } \\
\text { ID:172-204 }\end{array}$ & $\begin{array}{l}\text { CO:10 } \\
\text { ID:10 }\end{array}$ & 11 & $\begin{array}{l}\text { CO: } 0.88 \\
\text { ID: } 0.90\end{array}$ & $\begin{array}{l}\text { CO: } 0.84 \\
\text { ID: } 0.83\end{array}$ & $\begin{array}{l}\text { CO:0.000 } \\
\text { ID:0.000 }\end{array}$ \\
\hline
\end{tabular}

tGenBank Accession nos EU262763-EU262770. $N_{\mathrm{A}^{\prime}}$ mean number of alleles per locus; $H_{\mathrm{O}^{\prime}}$ observed and $H_{\mathrm{E}^{\prime}}$ expected heterozygosities; *, indicates significant deviation from Hardy-Weinberg Equilibrium after Bonferroni correction (Rice 1989). Null allele frequencies are based on Brookfield 2 estimates from Micro-Checker software; Sindicates significant evidence of null alleles with 95\% confidence intervals; and findicates significance with $99 \%$ confidence interval.

for all loci was an initial denaturation at $94^{\circ} \mathrm{C}$ for $2 \mathrm{~min}$ (B02, G07 and C02H08), 3 min (G12B11, H10E11 and E09B10) or $4 \mathrm{~min}$ (F09F10 and G02H10) followed by 35 cycles of $94{ }^{\circ} \mathrm{C}$ for $30 \mathrm{~s}$, annealing at $51^{\circ} \mathrm{C}$ (G12B11 and H10E11), $52{ }^{\circ} \mathrm{C}$ (E09B10, F09F10 and G02H10) or $55^{\circ} \mathrm{C}$ (B02, G07 and $\mathrm{C} 02 \mathrm{H} 08)$ for $45 \mathrm{~s}$, and extension at $72{ }^{\circ} \mathrm{C}$ for $45 \mathrm{~s}$. Cycling was followed with a 7-min extension at $72{ }^{\circ} \mathrm{C}(\mathrm{B} 02, \mathrm{G} 07$ and $\mathrm{C} 02 \mathrm{H} 08$ ) or a $30-\mathrm{min}$ extension at $60^{\circ} \mathrm{C}$. Of the 36 primer pairs that were designed and tested, eight pairs amplified and were variable in both populations.

PCR products were genotyped on an ABI 3130 genetic analyser and analysed with STRand software (Hughes 1998; Locke et al. 2000). Genotypic disequilibrium between pairs of loci was tested using FSTAT 2.9.3 (Goudet 2001). Hardy-Weinberg Equilibrium (HWE), number of alleles and expected and observed heterozygosities were estimated in Arlequin (Excoffier et al. 2005) and each locus was tested for null alleles using Micro-Checker (van Oosterhout et al. 2004). We found no evidence of linkage disequilibrium between loci. The number of alleles ranged from six to 20 per locus (Table 1). Two loci in each population demonstrated significant deviations from HWE (Table 1) after sequential Bonferroni correction (Rice 1989); however, only locus Coto_H10F_E11R violated HWE in each population. Moderate (0.05-0.20, Chapuis \& Estoup 2007) null allele frequencies were found at some loci (Table 1) which could be the result of a Wahlund effect or the presence of true null alleles, although we have no evidence of the latter since all individuals yielded amplification products (i.e. we found no null homozygotes). These eight new markers, plus previously characterized markers developed from other Vespertilionids (Piaggio et al. in press), now make it possible to undertake detailed population genetic studies of C. townsendii.

\section{Acknowledgements}

We are indebted to Kirk Navo, Lea Bonewell, and Mark Hayes for samples and support. We also wish to thank Jake Gratten for his helpful comments. A portion of this work was completed with the help of the Molecular Research Core Facility at Idaho State University. A.J.P. is supported by an Animal Plant Health Inspection Services Science Fellowship.

\section{References}

Burland TM, Barrrat EM, Racey PA (1998) Isolation and characterization of microsatellite loci in the brown long-eared bat, Plecotus auritus, and cross-species amplification within the family vespertilionidae. Molecular Ecology, 7, 133-140.

Castelo AT, Martins WS, Gao GR (2002) Tandem repeat occurrence locator. Bioinformatics, 8, 634-636.

Chapuis MP, Estoup A (2007) Microsatellite null alleles and estimation of population differentiation. Molecular Biology and Evolution, 24, 621-631.

Excoffier L, Laval G, Schneider S (2005) Arlequin version 3.0: an integrated software package for population genetics data analysis. Evolutionary Bioinformatics Online, 1, 47-50. 
Glenn TC, Schable NA (2005) Isolating microsatellite DNA loci. Methods in Enzymology, 395, 202-222.

Goudet J (2001) FSTAT, Version 2.9.3, A program to estimate and test gene diversities and fixation indices (updated from goudet 1995). University of Lausanne, Switzerland. Available from URL: http://www.unil.ch/izea/softwares/fstat.html.

Hughes S (1998) sтRand Nucleic Acid Analysis Software. Regents of the University of California, Davis, California. Available from URL: (http://www.vgl.ucdavis.edu/STRand ).

Locke M, Baack E, Toonen RJ (2000) The strand Manual. Available from URL: (http://www.vgl.ucdavis.edu/STRand/docs/ STRand_manual.pdf).

Martins W, de Sousa D, Proite K, Guimarães P, Moretzsohn M, Bertoli D (2006) New softwares for automated microsatellite marker development. Nucleic Acids Research, 34, E31.

van Oosterhout C, Hutchison WF, Wills DPM, Shipley P (2004) Micro-Checker: software for identifying and correcting genotyping errors in microsatellite data. Molecular Ecology Notes, 4, 535.

Piaggio AJ, Navo KW, Stihler CW (in press) Intraspecific comparison of population structure, genetic diversity, and dispersal among three subspecies of Townsend's big-eared bats, Corynorhinus townsendii townsendii, C. t. pallescens, and the endangered C. $t$. virginianus. Conservation Genetics. doi: 10.1007/s10592-008-9542-0.

Piaggio AJ, Perkins SL (2005) Molecular phylogeny of North
American long-eared bats (Vespertilionidae: corynorhinus): interand intraspecific relationships inferred from mitochondrial and nuclear DNA sequences. Molecular Phylogenetics and Evolution, 37, 762-775.

Pierson ED, Wackenhut MC, Altenbach JS et al. (1999) Species Conservation Assessment and Strategy for Townsend's Big-eared Bat (Corynorhinus townsendii townsendii and corynorhinus townsendii pallescens). Idaho Conservation Effort. Idaho Department of Fish and Game, Boise, Idaho.

Rice WR (1989) Analyzing tables of statistical tests. Evolution, 43, 223-225.

Rozen S, Skaletsky H (2000) Primer 3 on the WWW for general users and for biologist programmers. In: Bioinformatics Methods and Protocols: Methods in Molecular Biology (eds Krawetz S, Misener S), pp. 365-386. The Humana Press Inc., Totowa, New Jersey.

Staden R, Beal KF, Bonfield JK (1998) The Staden Package. In: Computer Methods in Molecular Biology, Bioinformatics Methods and Protocols (eds Misener S, Krawetz S), Vol. 132, pp. 115-130. The Humana Press Inc., Totowa, New Jersey.

Vonhof MJ, Davis CS, Fenton MB, Strobeck C (2002) Characterization of dinucleotide microsatellite loci in big brown bats (Eptesicus fuscus), and their use in other North American verspertilionid bats. Molecular Ecology Notes, 2, 167-170. 